\title{
Problemas de espaço dentário em adolescentes brasileiros e fatores associados
}

\author{
Dental spacing problems and associated factors \\ among Brazilian adolescents
}

Theodorico de Almeida Nunes Neto ${ }^{1}$

Erika Bárbara Abreu Fonseca Thomaz ${ }^{2}$

Meire Coelho Ferreira ${ }^{3}$

Alcione Miranda dos Santos ${ }^{2}$

Rejane Christine de Sousa Queiroz ${ }^{2}$

${ }^{1}$ Centro Universitário do Pará. Av. Nazaré 630, Nazaré. 66035-170 Belém PA Brasil.theo_neto@ hotmail.com

${ }^{2}$ Departamento de Saúde Pública, Centro de Ciências da Saúde, Universidade Federal do Maranhão.

${ }^{3}$ Programa de Mestrado em Odontologia, Universidade CEUMA.

\begin{abstract}
The scope of this study was to estimate the prevalence of dental spacing problems and associated factors among adolescents using data from the SB Brazil 2010 survey. The outcomes evaluated were dental spacing problems: space deficit (crowding and misalignment) and excess space (diastema and spacing) obtained using the DAI index. The association of independent variables with outcomes was assessed using a hierarchical model with four levels: contextual, socioeconomic and demographic characteristics, access to services and dental morbidity. Statistical analysis was performed using the chi-square test and univariate and multivariate Poisson distribution to estimate prevalence ratios (PR). The overall prevalence of space problems was $71.43 \%$, with misalignment being the most common type (56.4\%). The following aspects were significantly associated with excess space: age of 16, 18 and 19 years; being non-Caucasian $(P R=1.75)$, perception of speech problems $(P R=1.72)$ and periodontal pockets 4-5mm $(R P=1.56)$. For space deficit: family income up to 3 minimum wages, dental visit 1 year or more previously $(P R=1.19)$ and having one or more decayed teeth on average $(P R=1.32)$. There was a prevalence of spacing problems, especially with socioeconomic and demographic variables and morbidity as potential risk factors.
\end{abstract}

Key words Malocclusion, Orthodontics, Prevalence, Adolescents
Resumo O objetivo do estudo foi estimar a prevalência de problemas de espaço dentário em adolescentes brasileiros e fatores associados utilizando-se dados do inquérito SB Brasil 2010. Os desfechos avaliados foram os problemas de espaço dentário: déficit (apinhamento e desalinhamento) e excesso (diastema e espaçamento) obtidos pelo Índice de Estética Dental. A associação de variáveis independentes aos desfechos foi verificada a partir de um modelo hierarquizado em quatro níveis: contextuais, socioeconômicas e demográficas, acesso aos serviços e morbidade bucal. A análise estatística foi realizada utilizando-se teste quiquadrado, Regressão de Poisson uni e multivariada, estimando-se razões de prevalências (RP). A prevalência total de problemas de espaço foi de $71,43 \%$, sendo o desalinhamento o tipo mais comum (56,4\%). Associaram-se significativamente ao excesso de espaço: idade de 16, 18 e 19 anos, não ser branco $(R P=1,75)$, percepção de problemas na fala $(R P=1,72)$ e bolsa periodontal $4-5 \mathrm{~mm}$ $(R P=1,56)$. Para déficit de espaço: renda familiar até 3 salários mínimos, visita ao dentista há 1 ano ou mais $(R P=1,19)$ e ter em média um ou mais dentes cariados $(R P=1,32)$. Houve prevalência de problemas de espaço, destacando-se como potenciais fatores de risco variáveis socioeconômicas e sociodemográficas e de morbidade.

Palavras-chave Má oclusão, Ortodontia, Prevalência, Adolescentes 


\section{Introdução}

Dos três principais desafios atuais à odontologia mundial (doença periodontal, cárie dentária e má oclusão dos dentes), este último sempre esteve relacionado a problemas de acesso ao cuidado $^{1,2}$, com tratamentos onerosos e de longa duração.

Má oclusão refere-se a todos os desvios dentários e de maxila e mandíbula do alinhamento normal ${ }^{3}$, o que pode levar a alterações estéticas responsáveis por afetar negativamente a qualidade de vida dos indivíduos ${ }^{4}$.

No início da adolescência, a interação com a escola, com a comunidade e com os grupos de amigos exerce influência sobre comportamentos e valores ${ }^{5}$ dentro de um importante período de formação da autoestima. O estudo de Feu et al. ${ }^{6}$ aponta para melhor qualidade de vida entre adolescentes cujos tratamentos ortodônticos foram finalizados, comparados àqueles que ainda estão em tratamento, ou mesmo não o iniciaram.

Dada a forte ligação entre qualidade de vida e autoestima, percebe-se que a busca por tratamento ortodôntico visa, na grande maioria dos casos, à melhora desta ${ }^{7}$ e à diminuição de possíveis problemas psicossociais relacionados à aparência ${ }^{4,8}$.

Marques $^{9}$ assevera que $87,7 \%$ dos adolescentes eram desejosos de passar por um tratamento ortodôntico. Apontam ainda a preocupação dos pais com a estética bucal dos filhos como um dos principais fatores que levam à busca por este tipo de tratamento, bem como a insatisfação com a aparência dentofacial, recomendação do dentista e influência de colegas da escola que usam aparelho ortodôntico. Mas, apesar do princípio da integralidade ser assegurado pelo Sistema Único de Saúde e, ainda, a despeito de argumentos levantados na literatura ${ }^{1,10}$, o tratamento para as más oclusões ainda é pouco ofertado na rede pública do Brasil.

Dentre as várias formas de má oclusão dentária conhecidas, as relativas aos problemas de espaço e que afetam esteticamente o sorriso são o apinhamento, o espaçamento, o desalinhamento e o diastema, que acometem principalmente a região anterior de ambos os arcos dentários.

Até o momento não se conhecem estudos que abordem fatores relacionados ao excesso e ao déficit de espaço dentário com o uso de modelagem hierarquizada, sobretudo de base populacional e de abrangência nacional.

A abordagem hierarquizada representa um avanço em relação à modelagem de regressão convencional, que apresenta apenas um nível hierárquico, por permitir a inclusão de variáveis em diferentes níveis e, consequentemente, considerações relativas à ordenação temporal ou lógica entre os fatores e hipóteses de intermediação.

Desta forma, é importante investigar e intervir nos fatores que influenciam os problemas de espaço dentário na população de adolescentes no país, não apenas para o conhecimento dos profissionais no assunto, mas também porque pode contribuir tanto para a Política Nacional de Saúde Bucal quanto para a Política Nacional de Saúde do Adolescente no Brasil.

Assim, o objetivo do estudo foi avaliar a prevalência de problemas de espaço dentário e fatores associados em adolescentes brasileiros no ano 2010.

\section{Material e métodos}

Trata-se de uma pesquisa com dados secundários obtidos do Inquérito sobre as Condições de Saúde Bucal da População Brasileira, realizado em 2010 pelo Ministério da Saúde ("SB Brasil 2010"). Utilizaram-se os dados da amostra dos adolescentes na faixa etária de 15 a 19 anos, correspondendo a 5.367 participantes. Detalhes sobre o processo de cálculo e seleção da amostra podem ser acessados em publicação específica ${ }^{11}$. A pesquisa foi realizada em 177 municípios e teve representatividade para o Brasil, para as cinco regiões administrativas e para as capitais de estado e distrito federal.

Neste Inquérito foram coletados dados para diversos grupos etários (crianças, adolescentes, adultos e idosos), obtendo-se parâmetros clínicos por meio de exames odontológicos dos entrevistados, complementados por dados demográficos, socioeconômicos e de autoavaliação da saúde bucal. As equipes de campo foram constituídas por um examinador (cirurgião-dentista) e um anotador que participaram de oficinas para o treinamento dos exames bucais e calibração dos códigos e critérios de cada agravo bucal.

Características de má oclusão foram obtidas através do Índice de Estética Dental (DAI), sendo quatro delas relativas a problemas de espaço dentário e que são objeto do presente estudo: apinhamento e desalinhamento (problemas de déficit de espaço dentário); espaçamento e diastema (problemas de excesso de espaço dentário).

Foram estimadas prevalências expressas em porcentagem para cada um dos problemas de espaço (apinhamento, espaçamento, desalinha- 
mento e diastema), também para déficit de espaço (presença de apinhamento e/ou desalinhamento) e excesso de espaço (espaçamento e/ou diastema) e para o total de problemas de espaço (presença de um ou mais problemas). Para verificar a associação entre diastema, espaçamento, apinhamento e desalinhamento com as características geográficas, socioeconômicas e demográficas, de acesso aos serviços odontológicos e de morbidade bucal, foi utilizado o teste qui quadrado $(\mathrm{p}<0,05)$.

Para analisar os fatores explicativos dos desfechos (problemas de déficit e excesso de espaço dentário) foram realizadas análises de regressão de Poisson uni e multivariadas, com ajuste robusto, para atenuar possível subestimação de erro padrão, considerando-se que as variáveis dependentes são binárias e suas frequências (ou prevalências) foram superiores a 10\%. Estimaram-se razões de prevalências (RP) ajustadas e respectivos intervalos de confiança a 95\% (IC95\%). Variáveis independentes que alcançaram um valor de $\mathrm{p}<0,20$ na análise univariada foram selecionadas para o modelo multivariado. Permaneceram no modelo multivariado as variáveis independentes que tiveram valor de $\mathrm{p}<0,05$.

As variáveis explicativas foram consideradas a partir de um modelo hierarquizado ${ }^{12}$ e o referencial teórico para este estudo foi construído em quatro níveis. O primeiro nível incluiu as variáveis mais distais do modelo em relação à associação com os desfechos: região geopolítica (norte, nordeste, sul, sudeste e centro-oeste) e local de moradia (capital/distrito federal ou não capital) que foram assumidas como variáveis contextuais, tendo em vista o contexto geográfico e sociopolítico. O segundo nível incluiu variáveis relacionadas às condições socioeconômicas e demográficas que sofrem influências das variáveis contextuais e foram: sexo, idade $(15,16,17,18$ ou 19 anos), cor da pele (branco ou não branco), déficit escolar (com déficit e sem déficit), renda familiar em salários mínimos $(>3 ; 1-3$ ou $\leq 1)$, número de pessoas por cômodos (até 1 ou $>1$ ). O terceiro nível foi composto pelas variáveis relacionadas ao acesso aos serviços odontológicos e que são influenciadas pelas variáveis socioeconômicas e demográficas, sendo: tempo da última consulta odontológica ( $<1$ ano, $\geq 1$, ou nunca), local da última consulta odontológica (SUS ou não SUS). No quarto nível consideraram-se as variáveis relacionadas à morbidade bucal percebida e normativa, assumindo-se que essas são influenciadas pelo acesso aos serviços odontológicos: percepção de necessidade de tratamento (sim ou não), de problemas na fala ( (sim ou não), que afetavam os relacionamentos ( $\operatorname{sim}$ ou não), CPOD (CPOD $=0$ ou CPOD $\geq$ 1 ), dentes perdidos (nenhum-componente $\mathrm{P}$ do $\mathrm{CPOD}=0$ ou algum-componente $\mathrm{P}$ do $\mathrm{CPOD} \geq$ 1 ), dentes cariados (nenhum-componente $\mathrm{C}$ do $\mathrm{CPOD}=0$ ou algum-componente $\mathrm{C}$ do CPOD $\geq$ 1) e doença periodontal (nenhuma, sangramento, cálculo, bolsa de $4-5 \mathrm{~mm}$, bolsa $\geq 6 \mathrm{~mm}$ ).

As análises estatísticas foram realizadas no pacote estatístico Stata 11.0 (Stata Corp. 2009, College Station, Estados Unidos), utilizando-se os comandos "svy" que levam em consideração o efeito do desenho de amostra complexa e os pesos amostrais.

Em respeito ao que dispõe a Resolução 196/96 ${ }^{13}$ do Conselho Nacional de Saúde (CNS), os aspectos éticos da pesquisa foram atendidos pelo projeto do Inquérito SB Brasil 2010, que foi submetido e aprovado pelo Conselho Nacional de Ética Em Pesquisa (CONEP).

\section{Resultados}

A prevalência total de problemas de espaço dentário entre os adolescentes brasileiros foi de $71,43 \%$, sendo os problemas de déficit de espaço $(58,74 \%)$ maiores do que os de excesso de espaço dentário (18,98\%). O tipo de problema de espaço mais comum foi o desalinhamento dentário (56,37\%), seguido do apinhamento $(43,80 \%)$, espaçamento $(21,90 \%)$ e diastema $(15,07 \%)$.

A distribuição dos adolescentes brasileiros contendo espaçamento e diastemas (excesso de espaço dentário) bem como apinhamento e desalinhamento (déficit de espaço dentário), em relação às características geográficas, socioeconômicas e demográficas, de acesso aos serviços odontológicos e de morbidade bucal encontramse nas Tabelas 1 e 2 , respectivamente.

Verificou-se associação significativa do diastema com cor da pele $(\mathrm{p}<0,003)$, problemas na mastigação $(\mathrm{p}<0,044)$, problemas na fala $(\mathrm{p}<0,000)$ e problemas no relacionamento ( $\mathrm{p}$ $<0,001)$. O espaçamento dentário associou-se significativamente com a região geopolítica ( $\mathrm{p}<$ $0,027)$, cor da pele $(p<0,019)$ local de atendimento $(\mathrm{p}<0,033)$, problemas na fala $(\mathrm{p}<0,006)$ e doença periodontal $(\mathrm{p}<0,032)-($ Tabela 1$)$.

Verificou-se que o apinhamento dentário associou-se significativamente com renda familiar ( $\mathrm{p}<0,016)$, tempo decorrido desde a última consulta $(\mathrm{p}<0,026)$, necessidade de tratamento odontológico $(\mathrm{p}<0,001)$, problemas no relacio- 
Tabela 1. Distribuição dos problemas de excesso de espaço dentário em adolescentes brasileiros (diastema e espaçamento), segundo características geográficas, sociodemográficas, de uso dos serviços odontológicos e de morbidade bucal. Brasil, 2010.

\begin{tabular}{|c|c|c|c|c|c|c|c|c|c|c|}
\hline \multirow{3}{*}{ Variável } & \multicolumn{4}{|c|}{ Diastema } & \multirow{3}{*}{$\chi^{2}(\mathrm{p})$} & \multicolumn{4}{|c|}{ Espaçamento } & \multirow{3}{*}{$\chi^{2}(\mathbf{p})$} \\
\hline & \multicolumn{2}{|c|}{ Ausente } & \multicolumn{2}{|c|}{ Presente } & & \multicolumn{2}{|c|}{ Ausente } & \multicolumn{2}{|c|}{ Presente } & \\
\hline & $\mathbf{N}$ & $\%$ & $\mathbf{N}$ & $\%$ & & $\mathbf{N}$ & $\%$ & $\mathbf{N}$ & $\%$ & \\
\hline Brasil & 4.525 & 84,93 & 803 & 15,07 & & 4.180 & 78,10 & 1.172 & 21,90 & \\
\hline Região geopolítica & & & & & $0,2489(0,860)$ & & & & & $3,0063(0,027)$ \\
\hline Norte & 1.134 & 85,47 & 208 & 14,53 & & 1.036 & 73,30 & 305 & 26,70 & \\
\hline Nordeste & 1.159 & 85,26 & 232 & 14,74 & & 1.066 & 75,58 & 345 & 24,42 & \\
\hline Sudeste & 777 & 86,42 & 129 & 13,58 & & 733 & 81,73 & 176 & 18,27 & \\
\hline Sul & 701 & 86,50 & 107 & 13,50 & & 659 & 81,28 & 149 & 18,72 & \\
\hline Centro Oeste & 754 & 84,32 & 127 & 15,68 & & 686 & 76,20 & 197 & 23,80 & \\
\hline Local de moradia & & & & & $0,3550(0,551)$ & & & & & $0,0947(0,758)$ \\
\hline Capital/DF & 3.501 & 85,41 & 624 & 14,59 & & 3.236 & 80,28 & 901 & 19,72 & \\
\hline Outros & 1.024 & 86,33 & 179 & 13,67 & & 944 & 79,56 & 271 & 20,44 & \\
\hline Sexo & & & & & $0,0621(0,803)$ & & & & & $0,0690(0,793)$ \\
\hline Masculino & 2.042 & 85,74 & 401 & 14,26 & & 1.871 & 79,40 & 576 & 20,60 & \\
\hline Feminino & 2.483 & 86,35 & 402 & 13,65 & & 2.309 & 80,13 & 596 & 19,87 & \\
\hline Idade & & & & & $0,8634(0,476)$ & & & & & $0,6002(0,657)$ \\
\hline 15 & 1.202 & 84,33 & 228 & 15,67 & & 1.110 & 80,32 & 325 & 19,68 & \\
\hline 16 & 799 & 83,62 & 165 & 16,38 & & 737 & 75,52 & 233 & 24,48 & \\
\hline 17 & 846 & 89,72 & 126 & 10,28 & & 754 & 80,48 & 220 & 19,52 & \\
\hline 18 & 849 & 87,30 & 141 & 12,70 & & 800 & 81,90 & 193 & 18,10 & \\
\hline 19 & 829 & 86,31 & 803 & 13,69 & & 779 & 80,36 & 201 & 19,64 & \\
\hline Cor & & & & & $8,9599(0,003)$ & & & & & $5,5094(0,019)$ \\
\hline Branco & 1.889 & 90,35 & 278 & 9,65 & & 1.770 & 84,51 & 402 & 15,49 & \\
\hline Não branco & 2.636 & 82,40 & 525 & 17,60 & & 2.410 & 75,76 & 770 & 24,24 & \\
\hline Déficit escolar & & & & & $0,0040(0,950)$ & & & & & $0,6147(0,433)$ \\
\hline sem déficit & 2.456 & 86,00 & 410 & 14,00 & & 2.294 & 80,61 & 585 & 19,39 & \\
\hline com déficit & 2.069 & 86,13 & 393 & 13,87 & & 1.886 & 78,69 & 587 & 21,31 & \\
\hline Renda familiar & & & & & $0,3972(0,642)$ & & & & & $0,8025(0,432)$ \\
\hline$>3 \mathrm{SM}^{\mathrm{b}}$ & 1.353 & 87,36 & 227 & 12,64 & & 1.262 & 81,48 & 322 & 18,52 & \\
\hline 1 a $3 S^{b}$ & 2.194 & 85,03 & 406 & 14,97 & & 2.034 & 79,50 & 577 & 20,50 & \\
\hline$\leq 1 \mathrm{SM}^{\mathrm{b}}$ & 706 & 85,66 & 128 & 14,34 & & 642 & 76,24 & 200 & 23,76 & \\
\hline Pessoas/Cômodo & & & & & $0,7621(0,383)$ & & & & & $0,0005(0,982)$ \\
\hline até 1 & 1.254 & 84,87 & 237 & 15,13 & & 1.156 & 79,82 & 337 & 20,18 & \\
\hline$>1$ & 3.271 & 86,54 & 566 & 13,46 & & 3.024 & 79,76 & 835 & 20,24 & \\
\hline $\begin{array}{l}\text { Local do } \\
\text { atendimento }\end{array}$ & & & & & $2,2010(0,1383)$ & & & & & $4,5642(0,0329)$ \\
\hline SUS & 1.810 & 84,65 & 342 & 15,35 & & 1.668 & 76,96 & 499 & 23,04 & \\
\hline Não SUS & 2.095 & 88,10 & 313 & 11,90 & & 1.940 & 83,24 & 474 & 16,76 & \\
\hline $\begin{array}{l}\text { Tempo da última } \\
\text { consulta }\end{array}$ & & & & & $0,3584(0,690)$ & & & & & $0,5015(0,577)$ \\
\hline Nunca & 595 & 83,51 & 144 & 16,49 & & 2.107 & 75,99 & 550 & 24,01 & \\
\hline$<1$ ano & 2.287 & 86,82 & 363 & 13,18 & & 1.483 & 80,16 & 417 & 19,84 & \\
\hline$\geq 1$ ano & 1.598 & 85,94 & 290 & 14,06 & & 547 & 80,38 & 195 & 19,62 & \\
\hline $\begin{array}{l}\text { Necessita de } \\
\text { tratamento }\end{array}$ & & & & & $2,1047(0,147)$ & & & & & $1,9039(0,168)$ \\
\hline Sim & 2.972 & 85,25 & 566 & 14,75 & & 1.310 & 78,49 & 288 & 21,51 & \\
\hline Não & 1.392 & 89,20 & 204 & 10,80 & & 2.734 & 83,69 & 825 & 16,31 & \\
\hline
\end{tabular}




\begin{tabular}{|c|c|c|c|c|c|c|c|c|c|c|}
\hline \multirow{3}{*}{ Variável } & \multicolumn{4}{|c|}{ Diastema } & \multirow{3}{*}{$\chi^{2}(\mathbf{p})$} & \multicolumn{4}{|c|}{ Espaçamento } & \multirow{3}{*}{$\chi^{2}(\mathbf{p})$} \\
\hline & \multicolumn{2}{|c|}{ Ausente } & \multicolumn{2}{|c|}{ Presente } & & \multicolumn{2}{|c|}{ Ausente } & \multicolumn{2}{|c|}{ Presente } & \\
\hline & $\mathbf{N}$ & $\%$ & $\mathbf{N}$ & $\%$ & & $\mathbf{N}$ & $\%$ & $\mathbf{N}$ & $\%$ & \\
\hline $\begin{array}{l}\text { Problemas na } \\
\text { mastigação }\end{array}$ & & & & & $4,0544(0,044)$ & & & & & $2,5487(0,111)$ \\
\hline Não & 839 & 81,21 & 169 & 18,79 & & 3.401 & 80,81 & 919 & 19,19 & \\
\hline Sim & 3.670 & 87,29 & 630 & 12,71 & & 765 & 75,67 & 247 & 24,33 & \\
\hline Problemas na fala & & & & & $13,9464(0,000)$ & & & & & $7,6827(0,006)$ \\
\hline Sim & 249 & 69,30 & 62 & 30,70 & & 3.938 & 65,82 & 1.082 & 34,18 & \\
\hline Não & 4.264 & 87,14 & 738 & 12,86 & & 234 & 80,73 & 84 & 19,27 & \\
\hline $\begin{array}{l}\text { Problemas no } \\
\text { relacionamento }\end{array}$ & & & & & $10,5002(0,001)$ & & & & & $3,1910(0,074)$ \\
\hline Não & 3.960 & 87,46 & 674 & 12,54 & & 3.659 & 80,68 & 988 & 19,32 & \\
\hline Sim & 553 & 77,40 & 123 & 22,60 & & 511 & 74,61 & 176 & 25,39 & \\
\hline $\mathrm{CPOD}^{c}$ & & & & & $0,2926(0,589)$ & & & & & $0,2832(0,595)$ \\
\hline Não & 1.042 & 84,77 & 194 & 15,23 & & 963 & 81,10 & 275 & 18,90 & \\
\hline Sim & 3.483 & 86,47 & 609 & 13,53 & & 3.217 & 79,35 & 897 & 20,65 & \\
\hline $\begin{array}{l}\text { Dentes permanentes } \\
\text { perdidos }\end{array}$ & & & & & $0,6854(0,408)$ & & & & & $1,6113(0,205)$ \\
\hline Não & 3.612 & 85,75 & 633 & 14,25 & & 3.558 & 80,55 & 899 & 19,45 & \\
\hline Sim & 913 & 87,57 & 170 & 12,43 & & 822 & 76,08 & 273 & 23,92 & \\
\hline Dentes cariados & & & & & $0,7423(0,389)$ & & & & & $0,3439(0,558)$ \\
\hline Não & 2.110 & 85,12 & 350 & 14,88 & & 1.938 & 80,49 & 527 & 19,05 & \\
\hline Sim & 2.415 & 87,05 & 453 & 12,95 & & 2.242 & 79,01 & 645 & 20,99 & \\
\hline Doença periodontal & & & & & $0,7815(0,516)$ & & & & & $2,7490(0,037)$ \\
\hline Não & 2.114 & 86,44 & 364 & 13,56 & & 1.974 & 82,29 & 512 & 17,71 & \\
\hline Sangramento & 398 & 82,97 & 56 & 17,03 & & 358 & 80,97 & 96 & 19,03 & \\
\hline Cálculo & 1.461 & 87,14 & 263 & 12,86 & & 1.343 & 79,37 & 386 & 20,63 & \\
\hline bolsa $4-5 \mathrm{~mm}$ & 420 & 86,77 & 90 & 13,23 & & 387 & 72,43 & 127 & 27,57 & \\
\hline bolsa $\geq 6 \mathrm{~mm}$ & 27 & 67,16 & 5 & 32,84 & & 23 & 43,63 & 9 & 56,37 & \\
\hline
\end{tabular}

${ }^{\mathrm{a}} \mathrm{DF}$ - Distrito Federal. ${ }^{\mathrm{b}} \mathrm{SM}$ - salários mínimos. ${ }^{\mathrm{c}} \mathrm{CPOD}$ - Dentes Cariados, Perdidos e Obturados.

namento $(\mathrm{p}<0,035)$, ter em média um ou mais cariados, perdidos ou obturados $(\mathrm{p}<0,001)$, dentes cariados $(\mathrm{p}<0,018)$ e doença periodontal $(\mathrm{p}<0,000)$. O desalinhamento entre os adolescentes foi associado significativamente com renda familiar, necessidade de tratamento odontológico, ter em média um ou mais cariados, perdidos ou obturados, dente cariado e doença periodontal $(\mathrm{p}<0,000)-($ Tabela 2$)$.

Foram selecionadas para o modelo hierárquico multivariado dos problemas de excesso de espaço, as variáveis independentes: região geopolítica (nível 1), idade, cor da pele e déficit escolar (nível 2), local de atendimento da última consulta odontológica (nível 3), percepção da necessidade de tratamento, de problemas de mastigação, de fala, $C P O D \geq 1$, dentes permanentes perdidos e doença periodontal (nível 4). Permaneceram no modelo multivariado, região geopolítica, ida- de, cor, percepção de problemas na fala e doença periodontal (Tabela 3 ).

Para os problemas de déficit de espaço foram selecionadas as variáveis independentes: região geopolítica, local de moradia (nível 1), idade, renda familiar (nível 2), tempo da ultima consulta odontológica (nível 3), percepção da necessidade de tratamento odontológico, problemas na mastigação, problemas no relacionamento, CPOD $\geq$ 1, dentes cariados e doença periodontal (nível 4). Permaneceram no modelo multivariado renda familiar, tempo da ultima consulta odontológica, CPOD $\geq 1$ e doença periodontal (Tabela 4).

\section{Discussão}

Os resultados do presente estudo demonstraram que dentre os problemas de espaço dentário en- 
Tabela 2. Distribuição dos problemas de déficit de espaço dentário em adolescentes brasileiros (apinhamento e desalinhamento), segundo características geográficas, sociodemográficas, de uso dos serviços odontológicos e de morbidade bucal. Brasil, 2010.

\begin{tabular}{|c|c|c|c|c|c|c|c|c|c|c|}
\hline \multirow{3}{*}{ Variável } & \multicolumn{4}{|c|}{ Apinhamento } & \multirow{3}{*}{$\chi^{2}(\mathbf{p})$} & \multicolumn{4}{|c|}{ Desalinhamento } & \multirow{3}{*}{$\chi^{2}(\mathbf{p})$} \\
\hline & \multicolumn{2}{|c|}{ Ausente } & \multicolumn{2}{|c|}{ Presente } & & \multicolumn{2}{|c|}{ Ausente } & \multicolumn{2}{|c|}{ Presente } & \\
\hline & $\mathbf{N}$ & $\%$ & $\mathbf{N}$ & $\%$ & & $\mathrm{~N}$ & $\%$ & $\mathbf{N}$ & $\%$ & \\
\hline Brasil & 3.006 & 56,20 & 2.343 & 43,80 & & 2.334 & 43,63 & 3.016 & 56,37 & \\
\hline Região geopolítica & & & & & $0,2907(0,814)$ & & & & & $0,5294(0,656)$ \\
\hline Norte & 708 & 54,14 & 627 & 45,86 & & 540 & 36,00 & 802 & 64,00 & \\
\hline Nordeste & 810 & 54,43 & 603 & 45,57 & & 682 & 44,99 & 727 & 55,01 & \\
\hline Sudeste & 520 & 52,78 & 389 & 47,22 & & 395 & 43,28 & 514 & 56,72 & \\
\hline Sul & 571 & 53,89 & 337 & 46,1 & & 354 & 43,66 & 455 & 56,34 & \\
\hline Centro Oeste & 497 & 58,91 & 387 & 41,09 & & & 42,47 & & 57,53 & \\
\hline Local de moradia & & & & & $3,0763(0,080)$ & & & & & $2,8334(0,093)$ \\
\hline Capital/DF ${ }^{\mathrm{a}}$ & 2.349 & 58,42 & 1.784 & 41,58 & & 1.841 & 47,46 & 2.298 & 52,54 & \\
\hline Outros & 657 & 51,67 & 559 & 48,33 & & 493 & 40,83 & 718 & 59,17 & \\
\hline Sexo & & & & & $0,4315(0,511)$ & & & & & $0,5217(0,4703)$ \\
\hline Masculino & 1.346 & 52,49 & 1.099 & 47,51 & & 1.053 & 44,16 & 1.395 & 55,84 & \\
\hline Feminino & 1.660 & 54,83 & 1.244 & 45,17 & & 1.281 & 41,57 & 1.621 & 58,43 & \\
\hline Idade & & & & & $1,0765(0,366)$ & & & & & $2,5645(0,044)$ \\
\hline 15 & 796 & 49,82 & 637 & 50,18 & & 616 & 39,44 & 817 & 60,56 & \\
\hline 16 & 520 & 50,89 & 451 & 49,11 & & 365 & 32,27 & 604 & 65,73 & \\
\hline 17 & 543 & 53,39 & 430 & 46,61 & & 442 & 45,05 & 532 & 54,95 & \\
\hline 18 & 564 & 57,24 & 429 & 42,76 & & 447 & 47,22 & 532 & 52,78 & \\
\hline 19 & 583 & 58,49 & 396 & 41,51 & & 464 & 49,37 & 517 & 50,63 & \\
\hline Cor & & & & & $0,0072(0,932)$ & & & & & $0,0001(0,993)$ \\
\hline Branco & 1.258 & 53,87 & 913 & 46,13 & & 977 & 42,84 & 1.199 & 57,16 & \\
\hline Não branco & 1.748 & 53,55 & 1.430 & 46,45 & & 1.357 & 42,81 & 1.817 & 57,19 & \\
\hline Déficit escolar & & & & & $0,0482(0,826)$ & & & & & $0,3300(0,566)$ \\
\hline sem déficit & 1.638 & 54,01 & 1.241 & 45,99 & & 1.290 & 43,64 & 1.586 & 56,36 & \\
\hline com déficit & 1.368 & 53,29 & 1.102 & 46,71 & & 1.044 & 41,77 & 1.430 & 58,23 & \\
\hline Renda familiar & & & & & $4,4322(0,016)$ & & & & & $8,9758(0,000)$ \\
\hline$>3 \mathrm{SM}^{\mathrm{b}}$ & 969 & 61,92 & 614 & 38,08 & & 768 & 53,26 & 813 & 46,74 & \\
\hline 1 a $3 \mathrm{SM}^{\mathrm{b}}$ & 1.434 & 49,78 & 1.178 & 50,22 & & 1.079 & 38,03 & 1.532 & 61,97 & \\
\hline$\leq 1 \mathrm{SM}^{\mathrm{b}}$ & 427 & 48,48 & 412 & 51,52 & & 342 & 34,53 & 502 & 65,47 & \\
\hline Pessoas/Cômodo & & & & & $0,0984(0,754)$ & & & & & $0,4351(0,510)$ \\
\hline até 1 & 849 & 52,67 & 645 & 47,33 & & 665 & 40,87 & 827 & 59,13 & \\
\hline$>1$ & 2.157 & 54,11 & 1.698 & 45,89 & & 1.669 & 43,62 & 2.189 & 56,38 & \\
\hline $\begin{array}{l}\text { Local do } \\
\text { atendimento }\end{array}$ & & & & & $0,4547(0,500)$ & & & & & $1,2273(0,268)$ \\
\hline SUS & 1.153 & 51,31 & 1.014 & 48,69 & & 845 & 40,18 & 1.322 & 59,82 & \\
\hline Não SUS & 1.405 & 54,39 & 1.006 & 45,61 & & 1.117 & 44,79 & 1.295 & 55,21 & \\
\hline $\begin{array}{l}\text { Tempo da última } \\
\text { consulta }\end{array}$ & & & & & $3,6495(0,027)$ & & & & & $2,7427(0,068)$ \\
\hline Nunca & 1.595 & 57,81 & 1.061 & 42,19 & & 1.252 & 43,16 & 1.407 & 56,84 & \\
\hline$<1$ ano & 948 & 57,33 & 950 & 42,67 & & 700 & 47,25 & 1.196 & 52,75 & \\
\hline$\geq 1$ ano & 2.977 & 47,70 & 2.319 & 52,30 & & 359 & 36,96 & 383 & 63,04 & \\
\hline
\end{tabular}

continua

tre os adolescentes brasileiros, os déficits foram mais prevalentes $(58,74 \%)$ do que o excesso. Desalinhamento foi o tipo mais comum $(56,37 \%)$. Adolescentes com as menores rendas familiares e com deficiência no uso dos serviços de saúde bucal tiveram mais déficit de espaço dentário. $\mathrm{O}$ excesso de espaço aumentou com a idade, foi 75\% mais prevalente entre adolescentes não brancos, $72 \%$ maior entre aqueles com percepção de problemas na fala e $56 \%$ maior entre os portadores de bolsa periodontal de $4-5 \mathrm{~mm}$.

A maior prevalência do desalinhamento dentário corrobora com os achados de Tessarollo et al., em $2009^{14}$, com adolescentes entre 12 e 13 
Tabela 2. continuação

\begin{tabular}{|c|c|c|c|c|c|c|c|c|c|c|}
\hline \multirow{3}{*}{ Variável } & \multicolumn{4}{|c|}{ Apinhamento } & \multirow{3}{*}{$\chi^{2}(\mathbf{p})$} & \multicolumn{4}{|c|}{ Desalinhamento } & \multirow{3}{*}{$\chi^{2}(\mathbf{p})$} \\
\hline & \multicolumn{2}{|c|}{ Ausente } & \multicolumn{2}{|c|}{ Presente } & & \multicolumn{2}{|c|}{ Ausente } & \multicolumn{2}{|c|}{ Presente } & \\
\hline & $\mathrm{N}$ & $\%$ & $\mathbf{N}$ & $\%$ & & $\mathbf{N}$ & $\%$ & $\mathbf{N}$ & $\%$ & \\
\hline $\begin{array}{l}\text { Necessita de } \\
\text { tratamento }\end{array}$ & & & & & $9,9788(0,002)$ & & & & & $13,4059(0,000)$ \\
\hline Sim & 1.063 & 49,23 & 535 & 50,77 & & 878 & 37,35 & 722 & 62,65 & \\
\hline Não & 1.825 & 61,27 & 1.731 & 38,73 & & 1.373 & 51,73 & 2.183 & 48,27 & \\
\hline $\begin{array}{l}\text { Problemas na } \\
\text { mastigação }\end{array}$ & & & & & $0,3183(0,573)$ & & & & & $2,5327(0,112)$ \\
\hline Não & 2.471 & 54,28 & 1.848 & 45,72 & & 1.945 & 44,30 & 2.375 & 55,70 & \\
\hline Sim & 527 & 51,96 & 483 & 48,04 & & 384 & 37,68 & 626 & 62,32 & \\
\hline Problemas na fala & & & & & $2,9276(0,087)$ & & & & & $0,1684(0,682)$ \\
\hline Sim & 2.815 & 64,54 & 2.202 & 35,46 & & 2.195 & 40,24 & 2.821 & 59,76 & \\
\hline Não & 185 & 53,00 & 133 & 47,00 & & 134 & 43,01 & 185 & 56,99 & \\
\hline $\begin{array}{l}\text { Problemas no } \\
\text { relacionamento }\end{array}$ & & & & & $4,4605(0,0350)$ & & & & & $2,4659(0,117)$ \\
\hline Não & 2.695 & 54,92 & 1.949 & 45,08 & & 2.112 & 43,95 & 2.533 & 56,05 & \\
\hline Sim & 300 & 44,90 & 387 & 55,10 & & 214 & 35,36 & 473 & 64,64 & \\
\hline $\mathrm{CPOD}^{\mathrm{c}}$ & & & & & $12,3528(0,000)$ & & & & & $16,3820(0,000)$ \\
\hline Não & 762 & 64,29 & 475 & 35,71 & & 626 & 55,96 & 611 & 44,04 & \\
\hline Sim & 2.244 & 50,30 & 1.868 & 49,70 & & 1.708 & 38,62 & 2.405 & 61,38 & \\
\hline $\begin{array}{l}\text { Dentes } \\
\text { permanentes } \\
\text { perdidos }\end{array}$ & & & & & $0,4927(0,483)$ & & & & & $0,4857(0,486)$ \\
\hline Não & 2.384 & 53,18 & 1.871 & 46,82 & & 1.868 & 43,42 & 2.384 & 56,58 & \\
\hline Sim & 622 & 56,15 & 472 & 43,85 & & 466 & 39,98 & 632 & 60,02 & \\
\hline Dentes cariados & & & & & $5,6238(0,018)$ & & & & & $14,5861(0,000)$ \\
\hline Não & 1.516 & 58,14 & 949 & 41,86 & & 1.219 & 49,92 & 1.247 & 50,08 & \\
\hline Sim & 1.490 & 48,98 & 1.394 & 51,02 & & 1.115 & 35,27 & 1.769 & 64,73 & \\
\hline $\begin{array}{l}\text { Doença } \\
\text { periodontal }\end{array}$ & & & & & $6,3504(0,000)$ & & & & & $7,1441(0,000)$ \\
\hline Não & 1.547 & 60,28 & 937 & 39,72 & & 1.277 & 51,26 & 1.208 & 48,78 & \\
\hline sangramento & 219 & 35,32 & 234 & 64,68 & & 172 & 32,84 & 282 & 67,16 & \\
\hline Cálculo & 868 & 49,06 & 862 & 50,94 & & 633 & 34,90 & 1.096 & 65,10 & \\
\hline bolsa 4-5 mm & 279 & 47,51 & 236 & 52,49 & & 189 & 34,17 & 323 & 65,83 & \\
\hline bolsa $\geq 6 \mathrm{~mm}$ & 19 & 69,35 & 13 & 30,65 & & 9 & 49,05 & 23 & 50,95 & \\
\hline
\end{tabular}

${ }^{a}$ DF - Distrito Federal. ${ }^{\mathrm{b}} \mathrm{SM}$ - salários mínimos. ${ }^{\mathrm{c}} \mathrm{CPOD}$ - Dentes Cariados, Perdidos e Obturados.

anos, moradores de Balneário Camboriú (SC). Foi constatada uma prevalência de $65,9 \%$ no segmento superior e de $67,3 \%$ no inferior.

$\mathrm{O}$ apinhamento tem sido o problema de espaço dentário mais estudado ${ }^{15-20}$, sendo apontada por Proffit et al. ${ }^{21}$ como a má oclusão mais prevalente nos EUA entre 1989 e 1994 . O presente estudo encontrou uma prevalência de $43,8 \%$ de apinhamento dentário em adolescentes, maior que a encontrada na cidade de Moshi (Tanzânia) ${ }^{22} \mathrm{em}$ 2003, entre adolescentes de 12 a 15 anos (41,2\%) e também na Nigéria ${ }^{23}$ com pacientes entre $11 \mathrm{e}$ 18 anos $(21,6 \%)$. No entanto, foi menor do que os valores encontrados nos EUA $(56,2 \%)$ entre os adolescentes de 12 a 17 anos $^{21}$ e também na Jordânia que verificou tanto a prevalência de apinhamento mandibular (57\%) quanto maxilar $(44 \%)^{24}$ na faixa etária de 12 a 17 anos.

Prevalências inferiores ao do presente estudo foram encontradas na Colômbia em 2001 $(59,4 \%)^{25}$, no Kuwait em $2005(73,2 \%)^{26}$, na Índia em $2009(62,7 \%)^{27}$, na Arábia Saudita $(81,4 \%)^{28}$ para o apinhamento no segmento anteroinferior da arcada de mulheres de 13 a 15 anos de idade na Jordânia em $2005(50,4 \%)^{29}$ e com jovens de 16 a 18 anos na Finlândia $(68 \%)^{30}$.

Enquanto os problemas de excesso de espaço dentário (diastema e espaçamento) ficaram as- 
Tabela 3. Regressão de Poisson univariada e multivariada para problemas de excesso de espaço dentário em adolescentes brasileiros, de acordo com níveis hierárquicos. Brasil, 2010 ( $\mathrm{n}=5.367$ ).

\begin{tabular}{|c|c|c|c|c|c|c|c|c|}
\hline \multirow{3}{*}{ Variável } & \multicolumn{8}{|c|}{ Problemas de Excesso de Espaço } \\
\hline & \multicolumn{2}{|c|}{ Ausente } & \multicolumn{2}{|c|}{ Presente } & \multirow{2}{*}{$\mathbf{R P}^{*}(\mathrm{IC} 95 \%)$} & \multirow{2}{*}{$\mathrm{p}$ valor } & \multirow{2}{*}{$\mathrm{RP}^{* *}(\mathrm{IC} 95 \%)$} & \multirow{2}{*}{ p valor } \\
\hline & $\mathbf{N}$ & $\%$ & $\mathbf{N}$ & $\%$ & & & & \\
\hline \multicolumn{9}{|c|}{ NÍVEL 1 - Contextuais } \\
\hline \multicolumn{9}{|l|}{ Região geopolítica } \\
\hline Norte & 979 & 72,71 & 246 & 23,29 & 1 & & 1 & \\
\hline Nordeste & 985 & 78,57 & 280 & 21,43 & $0,92(0,67-1,26)$ & 0,604 & $0,92(0,67-1,26)$ & 0,604 \\
\hline Sudeste & 705 & 86,38 & 122 & 13,62 & $0,58(0,40-0,86)$ & 0,007 & $0,58(0,40-0,86)$ & 0,007 \\
\hline Sul & 633 & 82,17 & 127 & 17,83 & $0,77(0,53-1,11)$ & 0,155 & $0,77(0,53-1,11)$ & 0,155 \\
\hline Centro Oeste & 656 & 79,51 & 152 & 20,49 & $0,88(0,64-1,22)$ & 0,440 & $0,88(0,64-1,22)$ & 0,440 \\
\hline \multicolumn{9}{|l|}{ Local de moradia } \\
\hline Capital/DFa & 3.061 & 82,60 & 720 & 17,40 & 1 & & & \\
\hline Outros & 897 & 83,88 & 207 & 16,12 & $0,93(0,72-1,19)$ & 0,556 & & \\
\hline \multicolumn{9}{|c|}{$\begin{array}{l}\text { NÍVEL } 2 \text { - Socioeconômicas } \\
\text { e demográficas }\end{array}$} \\
\hline \multicolumn{9}{|l|}{ Sexo } \\
\hline Masculino & 1.770 & 83,82 & 451 & 16,18 & 1 & & & \\
\hline Feminino & 2.188 & 83,19 & 476 & 16,81 & $1,04(0,78-1,37)$ & 0,792 & & \\
\hline \multicolumn{9}{|l|}{ Idade } \\
\hline 15 & 1.056 & 89,20 & 234 & 10,80 & 1 & & 1 & \\
\hline 16 & 693 & 79,42 & 186 & 20,58 & $1,91(1,38-2,64)$ & 0,000 & $1,85(1,35-2,54)$ & 0,000 \\
\hline 17 & 722 & 83,63 & 182 & 16,37 & $1,52(0,96-2,40)$ & 0,076 & $1,49(0,95-2,33)$ & 0,081 \\
\hline 18 & 754 & 83,20 & 161 & 16,80 & $1,56(1,02-2,36)$ & 0,038 & $1,54(1,02-2,33)$ & 0,039 \\
\hline 19 & 733 & 80,09 & 164 & 19,91 & $1,84(1,14-3,00)$ & 0,013 & $1,81(1,34-2,88)$ & 0,012 \\
\hline \multicolumn{9}{|l|}{ Cor } \\
\hline Branco & 1.693 & 88,10 & 322 & 11,90 & 1 & & 1 & \\
\hline Não Branco & 2.265 & 79,41 & 605 & 20,59 & $1,73(1,27-2,35)$ & 0,001 & $1,75(1,23-2,48)$ & 0,002 \\
\hline \multicolumn{9}{|l|}{ Déficit Escolar } \\
\hline Sem déficit & 2.176 & 85,36 & 475 & 1464 & 1 & & & \\
\hline Com déficit & 1.782 & 81,11 & 452 & 1889 & $1,29(0,97-1,72)$ & 0,083 & & \\
\hline \multicolumn{9}{|l|}{ Renda Familiar } \\
\hline$>3 \mathrm{SM}^{\mathrm{b}}$ & 1.200 & 85,90 & 259 & 14,10 & 1 & & & \\
\hline 1 a $3 \mathrm{SM}^{\mathrm{b}}$ & 1.921 & 82,25 & 461 & 17,75 & $1,56(0,84-1,90)$ & 0,270 & & \\
\hline$\leq 1 \mathrm{SM}^{\mathrm{b}}$ & 606 & 81,50 & 149 & 18,50 & $1,31(0,86-2,01)$ & 0,212 & & \\
\hline \multicolumn{9}{|l|}{ Pessoas/cômodo } \\
\hline até 1 & 1.095 & 83,56 & 268 & 16,44 & 1 & & & \\
\hline$>1$ & 2.863 & 83,46 & 659 & 16,54 & $1,01(0,73-1,39)$ & 0,972 & & \\
\hline \multicolumn{9}{|c|}{$\begin{array}{l}\text { NÍVEL } 3 \text { - Acesso aos } \\
\text { serviços odontológicos }\end{array}$} \\
\hline \multicolumn{9}{|c|}{ Local de atendimento } \\
\hline SUS & 1.574 & 82,02 & 385 & 17,98 & 1 & & & \\
\hline Não SUS & 1.855 & 86,01 & 385 & 13,99 & $0,78(0,55-1,10)$ & 0,155 & & \\
\hline \multicolumn{9}{|c|}{ Tempo da última consulta } \\
\hline$<1$ ano & 2.021 & 66,45 & 429 & 33,55 & 1 & & & \\
\hline$\geq 1$ ano & 1.392 & 74,74 & 338 & 25,26 & $0,02(0,68-1,54)$ & 0,917 & & \\
\hline Nunca & 506 & 71,13 & 152 & 28,87 & $1,32(0,83-2,11)$ & 0,243 & & \\
\hline
\end{tabular}

continua

sociados significativamente com a cor da pele e problemas de fala, os problemas de déficit de espaço dentário (apinhamento e desalinhamento) foram associados com cor da pele, renda familiar, necessidade de tratamento, ter em média um ou mais dentes cariados, perdidos ou obturados, ter dentes cariados e doença periodontal.

A cor da pele ficou associada com todos os problemas de espaço dentário, que é uma característica fortemente relacionada a fatores genéticos, 
Tabela 3. continuação

\begin{tabular}{|c|c|c|c|c|c|c|c|c|}
\hline \multirow{3}{*}{ Variável } & \multicolumn{8}{|c|}{ Problemas de Excesso de Espaço } \\
\hline & \multicolumn{2}{|c|}{ Ausente } & \multicolumn{2}{|c|}{ Presente } & \multirow{2}{*}{$\mathrm{RP}^{*}(\mathrm{IC} 95 \%)$} & \multirow{2}{*}{ p valor } & \multirow{2}{*}{$\mathrm{RP}^{* *}(\mathrm{IC} 95 \%)$} & \multirow{2}{*}{ p valor } \\
\hline & $\mathbf{N}$ & $\%$ & $\mathbf{N}$ & $\%$ & & & & \\
\hline \multicolumn{9}{|c|}{$\begin{array}{l}\text { NÍVEL } 4 \text { - Condição de } \\
\text { saúde bucal percebida e } \\
\text { normativa }\end{array}$} \\
\hline \multicolumn{9}{|c|}{ Necessita de tratamento } \\
\hline Não & 1.242 & 85,29 & 248 & 14,71 & 1 & & & \\
\hline Sim & 1.588 & 83,30 & 630 & 16,70 & $1,14(076-1,69)$ & 0,532 & & \\
\hline \multicolumn{9}{|c|}{ Problemas na mastigação } \\
\hline Não & 3.216 & 84,44 & 732 & 15,56 & 1 & & & \\
\hline Sim & 728 & 79,64 & 192 & 20,36 & $1,31(0,88-1,94)$ & 0,179 & & \\
\hline \multicolumn{9}{|l|}{ Problemas na fala } \\
\hline Não & 3.738 & 84,43 & 861 & 15,57 & 1 & & 1 & \\
\hline Sim & 212 & 67,81 & 62 & 32,19 & $2,07(1,33-3,21)$ & 0,001 & $1,72(1,12-2,64)$ & 0,014 \\
\hline \multicolumn{9}{|l|}{$\begin{array}{l}\text { Problemas no } \\
\text { relacionamento }\end{array}$} \\
\hline Não & 3.476 & 84,03 & 796 & 15,97 & 1 & & & \\
\hline Sim & 473 & 80,78 & 124 & 19,22 & $1,20(0,84-1,73)$ & 0,318 & & \\
\hline \multicolumn{9}{|l|}{$\mathrm{CPOD}^{\mathrm{c}}$} \\
\hline Zero & 916 & 86,40 & 207 & 13,60 & 1 & & & \\
\hline $1 \mathrm{ou}+$ & 3.042 & 82,58 & 720 & 17,42 & $1,28(0,92-1,78)$ & 0,138 & & \\
\hline \multicolumn{9}{|l|}{ Dentes perdidos } \\
\hline Zero & 3.193 & 84,55 & 694 & 15,45 & 1 & & & \\
\hline $1 \mathrm{ou}+$ & 765 & 78,45 & 233 & 21,55 & $1,39(0,97-2,00)$ & 0,069 & & \\
\hline \multicolumn{9}{|l|}{ Dentes cariados } \\
\hline Zero & 1.851 & 84,55 & 416 & 15,45 & 1 & & & \\
\hline $1 \mathrm{ou}+$ & 2.107 & 82,37 & 511 & 16,51 & $1,14(0,86-1,52)$ & 0,361 & & \\
\hline \multicolumn{9}{|c|}{ Doença periodontal } \\
\hline Não & 1.882 & 86,12 & 400 & 13,88 & 1 & & 1 & \\
\hline Sangramento & 344 & 84,77 & 79 & 15,23 & $1,10(0,63-1,90)$ & 0,740 & $0,97(0,59-1,61)$ & 0,918 \\
\hline Cálculo & 1.270 & 82,38 & 308 & 17,62 & $1,27(0,90-1,80)$ & 0,180 & $1,12(0,80-1,59)$ & 0,505 \\
\hline Bolsa 4-5 mm & 357 & 74,34 & 105 & 25,66 & $1,85(1,22-2,80)$ & 0,004 & $1,56(1,03-2,37)$ & 0,036 \\
\hline Bolsa $\geq 6 \mathrm{~mm}$ & 22 & 52,07 & 6 & 47,93 & $3,75(1,63-8,64)$ & 0,002 & $2,36(0,92-6,03)$ & 0,074 \\
\hline
\end{tabular}

${ }^{*}$ Razão de Prevalência Não Ajustada. ${ }^{* *}$ Razão de Prevalência Ajustada. ${ }^{a}$ DF - Distrito Federal. ${ }^{b}$ SM - salários mínimos. ${ }^{c}$ CPOD - Dentes Cariados, Perdidos e Obturados.

muito comuns entre os problemas ortodônticos ${ }^{15}$. Outro fator que chama atenção é a percepção da necessidade de tratamento entre os adolescentes com déficit de espaço dentário, sugerindo que tanto o apinhamento quanto o desalinhamento na região anterior estão entre os problemas que causam insatisfação com a aparência ${ }^{31,32}$.

No presente estudo obteve-se prevalência de $21,9 \%$ para o espaçamento dentário, uma condição pouco estudada. Esse achado foi menor do que a encontrada por Dacosta ${ }^{23}$ na Nigéria, para espaçamentos no segmento anterossuperior $(30 \%)$, e anteroinferior $(45,9 \%)$. No entanto, maior que as encontradas na Hungria: 10,4\% e $2,9 \%$ respectivamente ${ }^{33}$.
Dentre todos os espaçamentos dentais estudados, talvez o diastema interincisivos centrais seja o que mais chama a atenção de Ortodontistas, clínicos e leigos, bem como o que mais incomoda esteticamente aqueles que o possuem, $\mathrm{e}$ é uma condição normal nas dentaduras decídua e mista, que tende a desaparecer com a irrupção dos caninos permanentes e segundos molares ${ }^{34}$. A prevalência encontrada nesse estudo $(15,1 \%)$ foi semelhante à de jovens militares americanos de 17 a 25 anos $(14,8 \%)^{35}$ na década de 1960 , porém bem maior que a de jovens húngaros $(7,8 \%) \mathrm{em}$ $2006^{33}$. No Brasil, um estudo ${ }^{36}$ que utilizou dados do SB Brasil 2003 encontrou uma prevalência de $6,7 \%$ na idade de 18 anos. 
Tabela 4. Regressão de Poisson univariada e multivariada para problemas de déficit de espaço dentário em adolescentes brasileiros, de acordo com níveis hierárquicos. Brasil, 2010 ( $\mathrm{n}=5.367)$.

\begin{tabular}{|c|c|c|c|c|c|c|c|c|}
\hline \multirow{3}{*}{ Variável } & \multicolumn{8}{|c|}{ Problemas de déficit de espaço dentário } \\
\hline & \multicolumn{2}{|c|}{ Ausente } & \multicolumn{2}{|c|}{ Presente } & \multirow{2}{*}{$\mathrm{RP}^{*}(\mathrm{IC} 95 \%)$} & \multirow{2}{*}{ p valor } & \multirow{2}{*}{$\mathrm{RP}^{* *}(\mathrm{IC} 95 \%)$} & \multirow{2}{*}{ p valor } \\
\hline & $\mathbf{N}$ & $\%$ & $\mathbf{N}$ & $\%$ & & & & \\
\hline \multicolumn{9}{|c|}{ NÍVEL 1 - Contextuais } \\
\hline \multicolumn{9}{|c|}{ Região geopolítica } \\
\hline Norte & 495 & 33,64 & 840 & 66,36 & 1 & & 1 & \\
\hline Nordeste & 643 & 42,03 & 764 & 57,97 & $0,87(0,73-1,00)$ & 0,055 & $0,90(0,79-1,04)$ & 0,147 \\
\hline Sudeste & 373 & 40,41 & 536 & 59,59 & $0,90(0,78-1,04)$ & 0,145 & $0,89(0,77-1,02)$ & 0,095 \\
\hline Sul & 345 & 42,02 & 462 & 57,98 & $0,87(0,75-1,02)$ & 0,083 & $0,86(0,74-1,00)$ & 0,049 \\
\hline Centro Oeste & 347 & 41,11 & 534 & 58,89 & $0,89(0,73-1,07)$ & 0,215 & $0,89(0,73-1,08)$ & 0,225 \\
\hline \multicolumn{9}{|l|}{ Local de moradia } \\
\hline Capital/DFa & 1.735 & 44,86 & 2.394 & 55,14 & 1 & & 1 & \\
\hline Não capital & 468 & 38,30 & 742 & 61,70 & $1,12(0,99-1,26)$ & 0,070 & $1,13(1,00-1,27)$ & 0,048 \\
\hline \multicolumn{9}{|c|}{$\begin{array}{l}\text { NÍVEL } 2 \text { - Socioeconômicas } \\
\text { e demográficas }\end{array}$} \\
\hline \multicolumn{9}{|l|}{ Sexo } \\
\hline Masculino & 988 & 40,91 & 1.454 & 59,09 & 1 & & 1 & \\
\hline Feminino & 1.215 & 39,67 & 1.682 & 60,33 & $1,02(0,91-1,15)$ & 0,736 & & \\
\hline \multicolumn{9}{|l|}{ Idade } \\
\hline 15 & 584 & 37,82 & 847 & 62,18 & 1 & & 1 & \\
\hline 16 & 348 & 31,46 & 621 & 68,54 & $1,10(0,95-1,29)$ & 0,227 & $1,10(0,94-1,29)$ & 0,214 \\
\hline 17 & 410 & 42,61 & 561 & 57,39 & $0,92(0,77-1,10)$ & 0,381 & $0,93(0,77-1,11)$ & 0,399 \\
\hline 18 & 419 & 42,87 & 573 & 57,13 & $0,92(0,78-1,09)$ & 0,325 & $0,92(0,78-1,08)$ & 0,317 \\
\hline 19 & 442 & 47,50 & 534 & 52,50 & $0,84(0,73-1,00)$ & 0,022 & $0,85(0,74-0,98)$ & 0,024 \\
\hline \multicolumn{9}{|l|}{ Cor } \\
\hline Não Branco & 923 & 40,73 & 1.247 & 59,27 & 1 & & & \\
\hline Branco & 1.280 & 39,88 & 1.889 & 60,12 & $1,01(0,90-1,15)$ & 0,826 & & \\
\hline \multicolumn{9}{|l|}{ Déficit Escolar } \\
\hline Sem déficit & 1.222 & 41,01 & 1.651 & 58,99 & 1 & & & \\
\hline Com déficit & 981 & 39,32 & 1.485 & 60,68 & $1,03(0,93-1,14)$ & 0,588 & & \\
\hline \multicolumn{9}{|l|}{ Renda Familiar } \\
\hline$>3 \mathrm{SM}^{\mathrm{b}}$ & 730 & 50,20 & 849 & 49,80 & 1 & & 1 & \\
\hline 1 a $3 S^{b}$ & 1.021 & 35,33 & 1.587 & 64,67 & $1,30(1,09-1,55)$ & 0,004 & $1,27(1,08-1,50)$ & 0,004 \\
\hline$\leq 1 \mathrm{SM}^{\mathrm{b}}$ & 314 & 33,27 & 524 & 66,73 & $1,34(1,09-1,64)$ & 0,005 & $1,29(1,07-1,56)$ & 0,008 \\
\hline \multicolumn{9}{|l|}{ Pessoas/cômodo } \\
\hline até 1 & 635 & 39,66 & 855 & 60,34 & 1 & & & \\
\hline$>1$ & 1.568 & 40,52 & 2.281 & 59,73 & $0,99(0,86-1,13)$ & 0,838 & & \\
\hline
\end{tabular}

$\mathrm{Na}$ análise hierarquizada, verificou-se que, no bloco mais distal, a variável região geopolítica foi indicador de proteção para a ocorrência de excesso de espaço dentário $(\mathrm{RP}=0,58$; $\mathrm{IC}=0,40$ $0,86)$. Portanto, moradores da região Sudeste tinham $42 \%$ menos excesso de espaço do que os da região Norte. Isso pode ser explicado por ser uma região com características geopolíticas favoráveis a melhor oferta e organização dos serviços odontológicos, o que pode minimizar a ocorrência desses problemas tanto pela prevenção quanto pelo tratamento desse agravo.
Em relação às variáveis socioeconômicas e demográficas (Bloco 2), a cor não-branca e ser mais jovem foram indicativos de risco para excesso de espaço. O resultado para a cor é apoiado por estudos com análises descritivas como o de Lamenha et al. ${ }^{37}$ que encontrou valor médio da largura dos diastemas mais elevado em negros do que em brancos ou pardos.

As variáveis relacionadas ao acesso aos serviços odontológicos inseridas no bloco 3 não foram associadas ao desfecho excesso de espaço dentário. No entanto, para o bloco mais proximal 
Tabela 4. continuação

Problemas de déficit de espaço dentário

\begin{tabular}{|c|c|c|c|c|c|c|c|c|}
\hline \multirow[t]{2}{*}{ Variável } & \multicolumn{2}{|c|}{ Ausente } & \multicolumn{2}{|c|}{ Presente } & \multirow{2}{*}{$\mathrm{RP}^{*}(\mathrm{IC} 95 \%)$} & \multirow[t]{2}{*}{ p valor } & \multirow{2}{*}{$\mathbf{R P}^{* *}(\mathrm{IC} 95 \%)$} & \multirow{2}{*}{ p valor } \\
\hline & $\mathrm{N}$ & $\%$ & $\mathrm{~N}$ & $\%$ & & & & \\
\hline \multirow{2}{*}{\multicolumn{9}{|c|}{$\begin{array}{l}\text { NÍVEL } 3 \text { - Acesso aos } \\
\text { serviços odontológicos }\end{array}$}} \\
\hline & & & & & & & & \\
\hline \multicolumn{9}{|c|}{ Local de atendimento } \\
\hline SUS & 801 & 38,26 & 1.362 & 61,74 & 1 & & & \\
\hline Não SUS & 1.062 & 41,83 & 1.344 & 58,17 & $0,94(0,82-1,08)$ & 0,395 & & \\
\hline \multicolumn{9}{|c|}{ Tempo da última consulta } \\
\hline$<1$ ano & 1.196 & 45,11 & 1.457 & 54,89 & 1 & & 1 & \\
\hline$\geq 1$ ano & 656 & 34,02 & 1.236 & 65,98 & $1,20(1,04-1,39)$ & 0,013 & $1,19(1,04-1,36)$ & 0,013 \\
\hline Nunca & 329 & 40,44 & 412 & 59,56 & $1,08(0,90-1,31)$ & 0,388 & $1,04(0,86-1,24)$ & 0,702 \\
\hline \multirow{3}{*}{\multicolumn{9}{|c|}{$\begin{array}{l}\text { NÍVEL } 4 \text { - Condição de } \\
\text { saúde bucal percebida e } \\
\text { normativa }\end{array}$}} \\
\hline & & & & & & & & \\
\hline & & & & & & & & \\
\hline \multicolumn{9}{|c|}{ Necessita de tratamento } \\
\hline Não & 830 & 47,90 & 766 & 52,10 & 1 & & & \\
\hline Sim & 1.296 & 35,81 & 2.253 & 64,19 & $1,23(1,09-1,40)$ & 0,001 & & \\
\hline \multicolumn{9}{|c|}{ Problemas na mastigação } \\
\hline Não & 1.836 & 41,55 & 2.478 & 58,45 & 1 & & & \\
\hline Sim & 362 & 35,83 & 643 & 64,17 & $1,10(0,97-1,25)$ & 0,154 & & \\
\hline \multicolumn{9}{|l|}{ Problemas na fala } \\
\hline Não & 2.073 & 40,38 & 2.935 & 59,62 & 1 & & & \\
\hline Sim & 126 & 38,93 & 191 & 61,07 & $1,02(0,83-1,27)$ & 0,826 & & \\
\hline \multirow{2}{*}{\multicolumn{9}{|c|}{$\begin{array}{l}\text { Problemas afetam } \\
\text { relacionamento }\end{array}$}} \\
\hline & & & & & & & & \\
\hline Não & 1.992 & 41,65 & 2.644 & 58,35 & 1 & & & \\
\hline Sim & 203 & 31,04 & 482 & 68,96 & $1,18(1,02-1,38)$ & 0,031 & & \\
\hline \multicolumn{9}{|l|}{$\mathrm{CPOD}^{\mathrm{c}}$} \\
\hline Zero & 598 & 54,10 & 637 & 45,90 & 1 & & 1 & \\
\hline $1 \mathrm{ou}+$ & 1.605 & 35,85 & 2.499 & 64,15 & $1,40(1,17-1,66)$ & 0,000 & $1,32(1,11-1,56)$ & 0,001 \\
\hline \multicolumn{9}{|l|}{ Dentes perdidos } \\
\hline Zero & 1.758 & 40,54 & 2.488 & 59,46 & 1 & & & \\
\hline $1 \mathrm{ou}+$ & 445 & 38,98 & 648 & 61,02 & $1,03(0,88-1,20)$ & 0,745 & & \\
\hline \multicolumn{9}{|l|}{ Dentes cariados } \\
\hline Zero & 1.165 & 47,31 & 1.297 & 52,69 & 1 & & & \\
\hline $1 \mathrm{ou}+$ & 1.038 & 32,78 & 1.839 & 67,22 & $1,28(1,11-1,46)$ & 0,001 & & \\
\hline \multicolumn{9}{|c|}{ Doença periodontal } \\
\hline Não & 1.210 & 48,49 & 1.269 & 51,51 & 1 & & 1 & \\
\hline Sangramento & 159 & 28,15 & 294 & 71,85 & $1,39(1,20-1,62)$ & $<0,000$ & $1,36(1,16-1,60)$ & $<0,000$ \\
\hline Cálculo & 597 & 32,84 & 1.132 & 67,16 & $1,30(1,12-1,52)$ & 0,001 & $1,29(1,12-1,49)$ & $<0,000$ \\
\hline Bolsa 4-5 mm & 179 & 33,13 & 330 & 66,87 & $1,30(1,07-1,57)$ & 0,006 & $1,21(1,00-1,45)$ & 0,046 \\
\hline Bolsa $\geq 6 \mathrm{~mm}$ & 9 & 49,05 & 23 & 50,95 & $0,99(0,50-1,97)$ & 0,795 & $0,87(0,50-1,97)$ & 0,075 \\
\hline
\end{tabular}

${ }^{*}$ Razão de Prevalência Não Ajustada. ${ }^{\text {* }}$ Razão de Prevalência Ajustada. ${ }^{a}$ DF - Distrito Federal. ${ }^{b}$ SM - salários mínimos. ${ }^{c}$ CPOD - Dentes Cariados, Perdidos e Obturados.

(condições de saúde bucal percebida e normativa), as variáveis percepção de problemas na fala $(\mathrm{RP}=0,02 ; \mathrm{IC}=1,23-2,48)$ e doença periodontal $(\mathrm{RP}=1,56 ; \mathrm{IC}=1,03-2,37)$ foram indicadores de risco e proteção, respectivamente, para excesso de espaço. Adolescentes com percepção de problemas na fala tinham $98 \%$ menos excesso de espaço do que os que não tinham essa percepção. Além disso, o excesso de espaço foi 1,56 vezes mais prevalente entre adolescentes com doença periodontal do que nos demais. O som emitido por determinados fonemas é fortemente influenciado pelo espaço entre os dentes ${ }^{38}$, o que chama atenção durante a fala e é claramente percebido pelo indivíduo. A presença de bolsa rasa (4 a $5 \mathrm{~mm}$ ) pode levar à migração dentária patológica ${ }^{39,40}$. 
Em relação ao desfecho déficit de espaço dentário, as variáveis do bloco mais distal não foram associadas. No bloco 2 (variáveis socioeconômicas e demográficas), a renda familiar de um a três salários mínimos $(\mathrm{RP}=1,27 ; \mathrm{IC}=1,08-1,50)$ e menor ou igual a um salário mínimo $(\mathrm{RP}=1,29$; $\mathrm{IC}=1,07-1,56)$ foram indicadoras de risco para o desfecho. Quanto menor a renda, maior foi o risco da ocorrência de déficit de espaço dentário, podendo ser explicada pela baixa condição econômica para o pagamento dos serviços odontológicos. Outros estudos não evidenciaram essa associação, como o de Bernabé e Flores-Mir ${ }^{41}$ que relacionaram status socioeconômico à necessidade de tratamento ortodôntico e o de Meira et al. ${ }^{42}$ à severidade das oclusopatias. Tais diferenças podem ser explicadas pelas variáveis desfechos distintas ou mesmo pelos métodos de análises.

No bloco 3 (variáveis de acesso aos serviços odontológicos), o maior tempo desde a última consulta ( $\mathrm{RP}=1,19$; IC $=1,04-1,36)$ foi associado a déficit de espaço dentário. Ter frequentado o dentista há um ano ou mais (uso eventual do serviço) quando comparado a menos de um ano (uso mais regular dos serviços) dificulta a detecção precoce de problemas bucais, como a cárie e a doença periodontal, que influenciam a ocorrência das más oclusões de excesso de espaço. É possível que estas pessoas sejam as mais mutiladas em tratamentos mais invasivos, como exodontias. No bloco mais proximal (condições de saúde bucal percebida e normativa), as variáveis CPOD ( $\mathrm{RP}=1,32$; $\mathrm{IC}=1,11-1,56)$, doença periodontal: sangramento $(\mathrm{RP}=1,36$; $\mathrm{IC}=$ 1,16-1,60) e cálculo ( $\mathrm{RP}=1,29 ; \mathrm{IC}=1,12-1,49)$ foram indicadoras de risco para a ocorrência de déficit de espaço. A ocorrência de apinhamento e/ou desalinhamento associados à cárie dentária têm sido citados na literatura ${ }^{43}$, embora haja controvérsias ${ }^{44}$. A doença periodontal foi associada tanto ao excesso quanto ao déficit de espaço dentário. No entanto, nota-se que a associação com o primeiro desfecho se deu em uma fase mais avançada da doença e com o segundo, nas fases iniciais da doença. O binômio apinhamento-higiene deficiente ${ }^{45}$ é apontado como agravante da doença periodontal.

A abordagem empregada no presente estudo, separando a investigação de fatores associados aos problemas de excesso e de déficit de espaço é importante, tendo em vista que estes apresentam características distintas. Estudos similares são desconhecidos até o momento na literatura. Destaca-se que são poucos os estudos ${ }^{41,42}$ que abordam fatores sociais relacionados a tais tipos de más-o- clusões. Além disso, o presente estudo utilizou um modelo de análise hierarquizada que leva em consideração o ajuste das variáveis confundidoras e mediadoras por oferecer melhor adequação das relações hierárquicas de determinação entre as variáveis estudadas. No entanto, como se trata de um estudo transversal vale ressaltar que uma de suas limitações é o fato de não ser possível afirmar a relação temporal entre os fatores associados e o desfecho. Para verificar melhor esta relação, estudos longitudinais poderão ser desenvolvidos. Por outro lado, este é um estudo de base populacional, com estimativas precisas e cujos resultados podem ser extrapolados para os adolescentes brasileiros na faixa etária de 15 a 19 anos.

Importantes avanços aconteceram na assistência odontológica nos últimos anos, como a implementação do Sistema Único de Saúde que ajudou na redução da desigualdade social relativa ao uso do serviço odontológico. Observou-se também a melhoria na quantidade e qualidade dos atendimentos, além de investimentos em pessoal, tecnologia e na própria atenção básica de saúde ${ }^{46-48}$.

No que concerne à atenção à saúde do adolescente, esta vem se tornando uma prioridade em muitos países, inclusive no Brasil, que possui uma política nacional da juventude que contempla diretrizes para a área da saúde. Porém a saúde bucal não tem recebido a atenção merecida.

Dentre os problemas bucais que afetam os adolescentes, os de espaço dentário, sobretudo os da região anterior, que comprometem a estética, podem levar a um impacto negativo na autoestima e na qualidade de vida ${ }^{5}$. Tais problemas possuem etiologia desconhecida, normalmente sendo atribuída a influência de vários fatores, notadamente a predisposição genética e os fatores ambientais $^{17-19}$.

Conclui-se que adolescentes brasileiros têm alta prevalência de problemas estéticos de espaçamento dentário. Variáveis socioeconômicas, demográficas e de morbidade destacam-se como potenciais fatores de risco para este agravo. Os resultados apontados poderão contribuir para a elaboração de medidas de saúde pública quer na prevenção ou no tratamento desse tipo de má oclusão, uma vez que diversos agravos bucais podem ser tratados na atenção primária evitando demandas para os níveis de maior complexidade e custo. Tais medidas podem favorecer os adolescentes brasileiros, contribuindo para a melhoria da qualidade de vida, com a redução de problemas de interação social, baixa autoestima e melhor inserção no mercado de trabalho. 
Os adolescentes brasileiros apresentaram uma elevada prevalência de má oclusão relacionada a problemas estéticos de espaço, sendo o desalinhamento aquele com maior prevalência, seguido, em sequência, por apinhamento, espaçamento e diastema.

No presente estudo, o nível das variáveis demográficas e o daquelas relacionas à condição bucal percebida e também normativa mostraram-se como potenciais indicadores de risco para ocorrência de problemas de excesso de espaço dentário.

Já para a ocorrência de problemas de déficit de espaço dentário, as variáveis dos quatro níveis
- contextuais, socioeconômicas/demográficas, acesso aos serviços odontológicos e condições de saúde bucal normativa - foram associadas como potenciais indicadoras de risco.

\section{Colaboradores}

TA Nunes Neto, EBAF Thomaz e RCS Queiroz, participaram de todas as etapas da pesquisa. MC Ferreira revisou o texto e AM Santos contribuiu nas análises estatísticas. RCS Queiroz orientou a condução da pesquisa.

\section{Referências}

1. Guzzo SC, Finkler M, Reibnitz Júnior C, Reibnitz MT Ortodontia preventiva e interceptativa na rede de atenção básica do SUS: perspectiva dos cirurgiões-dentistas da Prefeitura Municipal de Florianópolis, Brasil. Cien Saude Colet 2014; 19(2):449-460.

2. Gomes AMM, Thomaz EBAF, Alves MTSSB, Silva AAM, Silva RA. Fatores associados ao uso dos serviços de saúde bucal: estudo de base populacional em municípios do Maranhão, Brasil. Cien Saude Colet 2014; 19(2):629-640.

3. Pinto EM, Gondim PP, Lima NS. Análise Crítica dos diversos métodos de avaliação e registro das más oclusões. Revista Dental Press de Ortodontia e Ortopedia Facial. 2008;13(1):82-91.

4. Peres SH, Goya S, Cortellazzi KL, Ambrosano GM, Meneghim MC, Pereira AC. Self-perception and malocclusion and their relation to oral appearance and function. Cien Saude Colet 2011;16(10):4059-4066.

5. Mitre RMA. Labirinto de espelhos: formação da autoestima na infância e adolescência. Cad Saude Publica 2005; 21(2):657-657.

6. Feu D, Oliveira BH, Sales HX, Miguel JAM. Más-oclusões e seu impacto na qualidade de vida de adolescentes que buscam tratamento ortodôntico. Ortodontia SPO. 2008; 41(4):355-365.

7. Oliveira CM, Sheiham A. Orthodontic treatment and its impact on oral health-related quality of life in Brazilian adolescents. J Orthod 2004; 31(1):20-27.

8. Marques LS, Filogônio CA, Filogônio CB, Pereira LJ, Pordeus IA, Paiva SM, Ramos-Jorge ML. Aesthetic impact of malocclusion in the daily living of Brazilian adolescents. J Orthod 2009; 36(3):152-159.
9. Marques LS. Prevalência da maloclusão e necessidade de tratamento ortodôntico em escolares de 10 a 14 anos de idade em Belo Horizonte, Minas Gerais, Brasil: enfoque psicossocial. Cad Saude Publica 2005; 21(4):1099-1106.

10. Hebling SRF, Pereira AC, Hebling E, Meneghim MC. Considerações para elaboração de protocolo de assistência ortodôntica em saúde coletiva. Cien Saude Colet 2007; 12(4):1067-1078.

11. Brasil. Ministério da Saúde (MS). Secretaria de Atenção à Saúde. Departamento de Atenção Básica. Projeto $S B$ Brasil 2010: Projeto Técnico. Brasília: MS; 2009.

12. Victora CG, Huttly SR, Fuchs SC, Olinto MT. The role of conceptual frameworks in epidemiological analysis: a hierarchical approach. Int J Epidemiol 1997; 26(1):224-227.

13. Brasil. Ministério da Saúde (MS). Conselho Nacional de Saúde. Resolução no 196 de 10 de outubro de 1996. Diretrizes e Normas Regulamentadoras de Pesquisas Envolvendo Seres Humanos. Diário Oficial da União 1996; 16 out.

14. Tessarollo FR, Feldens CA, Closs LQ. The impact of malocclusion on adolescents' dissatisfaction with dental appearance and oral functions. Angle Orthod 2012; 82(3):403-409.

15. Ting TY, Wong RW, Rabie AB. Analysis of genetic polymorphisms in skeletal Class I crowding. Am J Orthod Dentofacial Orthop 2011; 140(1):e9-15.

16. Pachì $\mathrm{F}$, Turlà R, Checchi AP. Head posture and lower arch dental crowding. Angle Orthod 2009; 79(5):873879 . 
17. Uysal T, Yagci A, Ozer T, Veli I, Ozturk A. Mandibular anterior bony support and incisor crowding: Is there a relationship? Am J Orthod Dentofacial Orthop 2012; 142(5):645-653.

18. Hasegawa Y, Terada K, Kageyama I, Tsukada S, Uzuka S, Nakahara R, Nakahara S. Influence of shovel-shaped incisors on the dental arch crowding in Mongolian females. Okajimas Folia Anat Jpn 2009; 86(2):67-72.

19. Shigenobu N, Hisano M, Shima S, Matsubara N, Soma K. Patterns of dental crowding in the lower arch and contributing factors. A statistical study. Angle Orthod 2007; 77(2):303-310.

20. Martins PP, Freitas MR, Freitas KMS, Canuto LFG, Janson GRP, Henriques JFC, Pinzan A. Apinhamento ântero-superior: revisão e análise crítica da literatura. Revista Dental Press de Ortodontia e Ortopedia Facial 2007; 12(2):105-114.

21. Proffit WR, Fields HW Junior, Moray LJ. Prevalence of malocclusion and orthodontic treatment need in the United States: estimates from the NHANES III survey. Int J Adult Orthodon Orthognath Surg 1998; 13(2):97106.

22. Rwakatema DS, Ng'ang'a PM, Kemoli AM. Orthodontic treatment needs among 12-15 year-olds in Moshi, Tanzania. East Afr Med J 2007; 84(5):226-232.

23. Dacosta OO. The prevalence of malocclusion among a population of northern Nigeria school children. West Afr J Med 1999; 18(2):91-96.

24. Hassan AM, Hani TD, Ayman HN. Frequency of Malocclusion in an Orthodontically Referred Jordanian Population. JRMS 2010; 17(4):19-23.

25. Thilander B, Pena L, Infante C, Parada SS, de Mayorga C. Prevalence of malocclusion and orthodontic treatment need in children and adolescents in Bogota, Colombia. An epidemiological study related to different stages of dental development. Eur J Orthod 2001; 23(2):153-167.

26. Behbehani F, Artun J, Al-Jame B, Kerosuo H. Prevalence and severity of malocclusion in adolescent Kuwaitis. Med Princ Pract 2005; 14(6):390-395.

27. Bhardwaj VK, Veeresha KL, Sharma KR. Prevalence of malocclusion and orthodontic treatment needs among 16 and 17 year-old school-going children in Shimla city, Himachal Pradesh. Indian J Dent Res 2011; 22(4):556-560.

28. Fadia M. Al Hummayani. Prevalence of Incisors Crowding in Saudi Arabian Female Students. Cairo Dental Journal 2004; 20(3):413-416

29. Abu Alhaija ES, Al-Khateeb SN, Al-Nimri KS. Prevalence of malocclusion in 13-15 year-old North Jordanian school children. Community Dent Health 2005; 22(4):266-271

30. Pietilä I, Pietilä T, Svedström-Oristo AL, Varrela J, Alanen P. Orthodontic treatment practices in Finnish municipal health centres with differing timing of treatment. Eur J Orthod 2009; 31(3):287-293.

31. Freitas MR, Freitas DS, Pinheiro FHSL, Freitas KMS. Prevalência das más oclusões em pacientes inscritos para tratamento ortodôntico na Faculdade de Odontologia de Bauru/USP. Revista da Faculdade de Odontologia de Bauru 2002; 10(3):164-169.

32. Peres KG, Traebert ESA, Marcenes W. Diferenças entre autopercepção e critérios normativos na identificação das oclusopatias. Rev Saude Publica 2002; 36(2):230-236.
33. Gábris K, Márton S, Madléna M. Prevalence of malocclusions in Hungarian adolescents. Eur J Orthod 2006; 28(5):467-470

34. Díaz ME. Diastema medio interincisal y su relación con el frenillo labial superior: una revisión. Rev Estomatol Herediana 2004; 14(1-2):95-100

35. Keene HJ. Distribution of diastemas in the dentition of man. Am J Phys Anthropol 1963; 21(4):437-441.

36. Traebert ES, Peres MA. Prevalence of malocclusions and their impact on the quality of life of 18-year-old young male adults of Florianópolis, Brazil. Oral Health Prev Dent 2005; 3(4):217-224

37. Lamenha EGR, Guimarães RP, Vicente Silva CH. Diastema mediano superior: aspectos etiológicos. International Journal Of Dentistry. 2007; 6(1):2-6

38. Koora K, Muthu MS, Rathna PV. Spontaneous closure of midline diastema following frenectomy. J Indian Soc Pedod Prev Dent 2007; 25(1):23-26

39. Costa MR, Silvério KG, Rossa CJ, Cirelli JA. Periodontal conditions of teeth presenting pathologic migration. Braz Oral Res 2004; 18(4):301-305.

40. Brunsvold MA. Pathologic tooth migration. J Periodontol 2005; 76(6):859-866

41. Bernabé E, Flores-Mir C. Orthodontic treatment need in Peruvian young adults evaluated through dental aesthetic index. Angle Orthod 2006; 76(3):417-421

42. Meira AC, Oliveira, MC, Aves TD. Severidade das oclusopatias e fatores associados em escolares de 12 anos no município de Feira de Santana, Bahia, 2009. Rev. Baiana de Saúde Pública 2011; 35(1):196-210.

43. Buczkowska-Radlinska J, Szyszka-Sommerfeld L, Wozniak K. Anterior tooth crowding and prevalence of dental caries in children in Szczecin, Poland. Community Dent Health 2012; 29(2):168-172.

44. Hafez HS, Shaarawy SM, Al-Sakiti AA, Mostafa YA. Dental crowding as a caries risk factor: a systematic review. Am J Orthod Dentofacial Orthop. 2012; 142(4):443-450.

45. Studen-Pavlovich D, Ranalli DN. Periodontal and soft tissue prevention strategies for the adolescent dental patient. Dent Clin North Am 2006; 50(1):51-67.

46. Paim J, Travassos C, Almeida C, Bahia L, Macinko J. O sistema de saúde brasileiro: história, avanços e desafios. The Lancet.com 2011; maio:11-31.

47. Mattos GCM, Ferreira EF, Leite ICG, Greco RM. A inclusão da equipe de saúde bucal na Estratégia Saúde da Família: entraves, avanços e desafios. Cien Saude Colet 2014; 19(2):373-382.

48. Mello ALSF, Andrade SR, Moyses SJ, Erdmann AL. Saúde bucal na rede de atenção e processo de regionalização. Cien Saude Colet 2014; 19(1):205-214.

Artigo apresentado em 03/08/2013

Aprovado em 27/08/2013

Versão final apresentada em 03/09/2013 